\title{
Unique cardiac magnetic resonance imaging findings of progressing myocardial damage in a patient with polymyositis
}

\author{
Noboru Kitamura $^{1}\left(\mathbb{D}\right.$, Hitomi Kobayashi $^{1}\left(\mathbb{D}\right.$, Masami $^{T^{2}}$ Takei $^{1}\left(\mathbb{D}\right.$, Yasuyuki Kobayashi $^{2}(\mathbb{D}$ \\ ${ }^{1}$ Department of Medicine, Division of Hematology and Rheumatology, Nihon University School of Medicine, Tokyo, Japan \\ ${ }^{2}$ Department of Medical Information and Communication Technology Research,St. Marianna University School of Medicine, Kanagawa, Japan
}

A 42-year-old female patient admitted to our hospital with muscular weakness and palpitations. Skeletal muscle biopsy reveled infiltration of lymphocytes surrounding muscle fibers consistent with the diagnosis of polymyositis (PM). Conventional cardiac angiography showed absence of macrovascular stenosis. The histopathological finding of myocardial biopsy was consistent with acute myocarditis. We performed cardiac magnetic resonance imaging (CMRI) to evaluate myocardial damage. Images were assessed for late gadolinium enhancement (LGE), T2-weighted imaging (T2WI) and extracellular volume (ECV) fraction. LGE images showed patchy enhancement in the septum and curvilinear myocardial enhancement in the inferolateral wall (Figure 1a). CMRI one year later revealed not significantly changed LGE (Figure 1b), expansion three years later (Figure 1c) and more expansion five years later (Figure 1d) without creatinine kinase elevation and cardiac symptoms. After five years, no significant abnormalities were seen in T2WI (Figure 2a). ECV fraction increased in the myocardial layer of anterolateral wall, the middle layer of septum and the papillary muscle, consistent with the region of LGE (Figure $2 b, c$ ).
ECV can detect diffuse myocardial edema in areas that do not exhibit abnormalities on T2WI. Presumably, this is active inflammatory cell
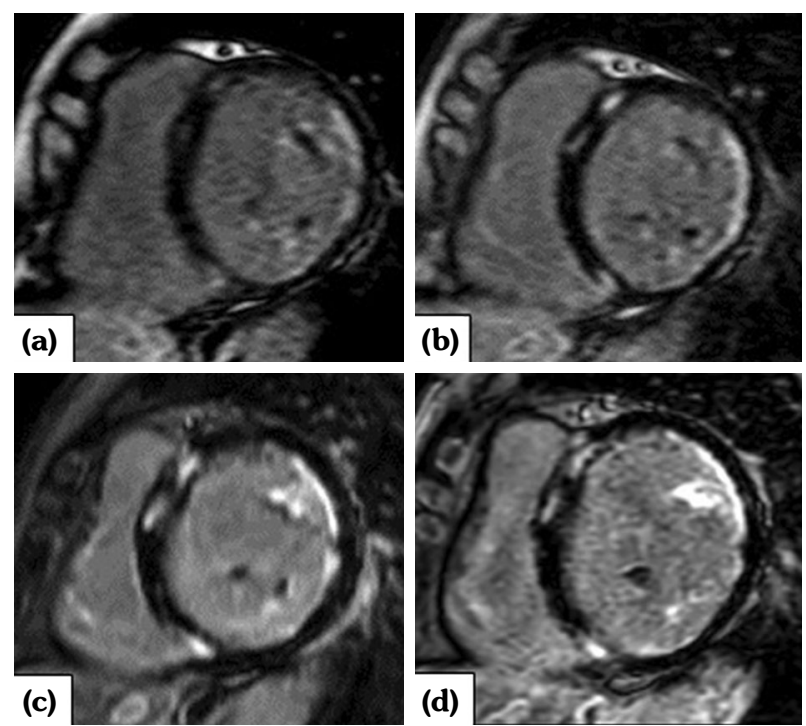

Figure 1. Progressing process of late gadolinium enhancement images. Late gadolinium enhancement (LGE) image showed patchy enhancements in septum and curvilinear submyocardial enhancement in inferolateral wall. LGE has progressed over time. (a) Onset; (b) One year later; (c) Three years later; (d) Five years later.

Received: September 02, 2020 Accepted: September 18, 2020 Published online: December 10, 2020

Correspondence: Noboru Kitamura, MD. Department of Medicine, Division of Hematology and Rheumatology, Nihon University School of Medicine, 173-8610 Tokyo, Japan. Tel: +81, 3-3972-8111 e-mail: kitamura.noboru@nihon-u.ac.jp

Kitamura N, Kobayashi H, Takei M, Kobayashi Y. Unique cardiac magnetic resonance imaging findings of progressing myocardial damage in a patient with polymyositis. Arch Rheumatol 2021;36(2):314-315. 

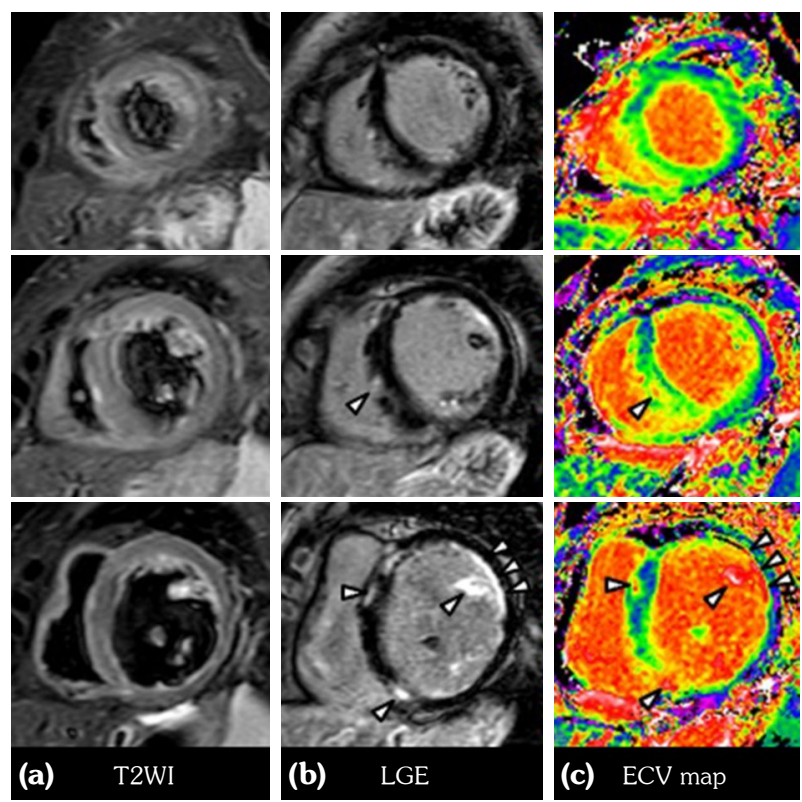

Figure 2. Cardiac magnetic resonance imaging five years after onset. (a) No significant abnormalities are seen in T2-weighted imaging; $(\mathbf{b}, \mathbf{c})$ Extracellular volume fraction increased in myocardial layer of anterolateral wall, middle layer of septum and papillary muscle, consistent with region of late gadolinium enhancement (white arrowheads).

T2WI: T2-weighted image; LGE: Late gadolinium enhancement; ECV map: Extracellular volume mapping. infiltration. Myocarditis in PM with such a unique LGE pattern is rare. There are a few reports of ECV increasing heterogeneously in PM. Cardiac involvement in PM is a frequent complication and often life-threatening. ${ }^{1,2}$ Despite alleviating inflammation and dissipation of symptoms, asymptomatic myocardial damage progressed gradually in this case. CMRI including ECV can be a useful method of evaluating myocardial damage.

\section{Declaration of conflicting interests}

The authors declared no conflicts of interest with respect to the authorship and/or publication of this article.

\section{Funding}

The authors received no financial support for the research and/or authorship of this article.

\section{REFERENCES}

1. Lundberg IE. The heart in dermatomyositis and polymyositis. Rheumatology (Oxford) 2006;45 Suppl 4:iv18-21.

2. Mavrogeni S, Douskou M, Manoussakis MN. Contrast-enhanced CMR imaging reveals myocardial involvement in idiopathic inflammatory myopathy without cardiac manifestations. JACC Cardiovasc Imaging 2011;4:1324-5. 